\title{
Article
}

\section{EBP50 suppresses the proliferation of MCF-7 human breast cancer cells via promoting Beclin-1/p62- mediated lysosomal degradation of c-Myc}

\author{
Hong LIU ${ }^{1}$, Wu-li ZHAO ${ }^{1}$, Jia-ping WANG ${ }^{2}$, Bing-mu XIN², Rong-guang SHAO ${ }^{1, *}$ \\ ${ }^{1}$ Key Laboratory of Biotechnology of Antibiotics of National Health and Family Planning Commission (NHFPC), Department of Oncology, \\ Institute of Medicinal Biotechnology, Peking Union Medical College, Chinese Academy of Medical Sciences, Beijing 100050, China; \\ ${ }^{2}$ China Astronaut Research and Training Centre, Beijing 100094, China
}

\begin{abstract}
c-Myc, a key activator of cell proliferation and angiogenesis, promotes the development and progression of breast cancer. Ezrin-radixin-moesin-binding phosphoprotein-50 (EBP50) is a multifunctional scaffold protein that suppresses the proliferation of breast cancer cells. In this study we investigated whether the cancer-suppressing effects of EBP50 resulted from its regulation of c-Myc signaling in human breast cancer MCF-7 cells in vitro and in vivo. We first found a significant correlation between EBP50 and C-Myc expression levels in breast cancer tissue, and demonstrated that EBP50 suppressed cell proliferation through decreasing the expression of C-Myc and its downstream proteins cyclin A, E and Cdc25A in MCF-7 cells. We further showed that EBP50 did not regulate c-Myc mRNA expression, but it promoted the degradation of c-Myc through the autophagic lysosomal pathway. Moreover, EBP50 promoted integration between c-Myc and p62, an autophagic cargo protein, triggering the autophagic lysosomal degradation of c-Myc. In EBP50-silenced MCF-7 cells, activation of autophagy by Beclin-1 promoted the degradation of c-Myc and inhibited cell proliferation. These results demonstrate that the EBP50/Beclin-1/p62/c-Myc signaling pathway plays a role in the proliferation in MCF-7 breast cancer cells: EBP50 stimulates the autophagic lysosomal degradation of c-Myc, thereby inhibits the proliferation of MCF-7 cells. Based on our results, promoting the lysosomal degradation of c-Myc might be a promising new strategy for treating breast cancer.
\end{abstract}

Keywords: human breast cancer; MCF-7 cells; c-Myc signaling; protein stability; protein interaction; autophagy lysosomal degradation

Acta Pharmacologica Sinica (2018) 39: 1347-1358; doi: 10.1038/aps.2017.171; published online 28 Dec 2017

\section{Introduction}

Ezrin-radixin-moesin (ERM)-binding phosphoprotein-50 (EBP50) belongs to the $\mathrm{Na}^{+} / \mathrm{H}^{+}$exchanger regulatory factor (NHERF) family. Members of this family typically contain two PDZ domains and a unique C-terminal domain, and act as multifunctional scaffold proteins to regulate cellular activities. EBP50 has multiple functions, including acting as a signaling molecule, junction protein, receptor, and transporter, and many are involved in cancer signaling ${ }^{[1,2]}$. EBP50 suppresses the proliferation of MDA-MB-231 breast cancer cells ${ }^{[3]}$, whereas EBP50 knockdown induces the growth of both MCF-7 and T47D cells ${ }^{[4]}$. Therefore, EBP50 has potential cancer-suppressive effects in breast cancer. However, the precise regulatory mechanism and signaling pathway used by EBP50 to regulate the proliferation of breast cancer cells remain unknown.

\footnotetext{
${ }^{*}$ To whom correspondence should be addressed.

E-mail shaor@imb.pumc.edu.cn

Received 2017-07-21 Accepted 2017-11-08
}

c-Myc is a key activator of cell proliferation and angiogenesis and promotes the development and progression of breast cancer $^{[5]}$. c-Myc transfers from cytoplasm to nucleus and later promotes cell cycle by activating the transcription progression of downstream molecules such as cyclin A1, cyclin E1, and cell division cycle 25 homolog A (Cdc25A) to drive cell proliferation $^{[6,7]}$. Moreover, c-Myc also promotes cell proliferation by up-regulating DNA synthesis ${ }^{[8]}$, transcription, mitochondrial biogenesis and protein biosynthesis ${ }^{[9]}$. Previously, we found that EBP50 increases the expression of PTEN in breast cancer cells $^{[2]}$ and that loss of PTEN causes c-Myc-initiated carcinogenesis $^{[10,11]}$, which suggests that EBP50 might suppress cell proliferation by regulating c-Myc signaling in breast cancer cells.

In this study, we showed that EBP50 inhibits the proliferation of MCF-7 breast cancer cells by stimulating the autophagic lysosomal degradation of c-Myc. EBP50 suppresses cell proliferation by decreasing c-Myc expression as well as reducing the levels of c-Myc transcription products. Moreover, EBP50 
promotes integration between c-Myc and p62, an autophagic cargo protein, to trigger autophagic lysosomal degradation of c-Myc. This activation of autophagy promotes the degradation of c-Myc and restrains cell proliferation in EBP50-silenced cells. Our findings provide insight into the molecular mechanism used by EBP50 to suppress cancer cell proliferation and reveal a novel signaling pathway for EBP50-inhibited tumor proliferation in breast cancer.

\section{Materials and methods}

\section{Reagents and antibodies}

CHX (01810), MG132 (C2211) and anti-SQSTM1 (P0067) antibodies were obtained from Sigma. Anti-human EBP50 (3394), c-Myc (13987), Cdc25A (3652), Cyclin E1 (20808), HA (3724), Myc (2278), Flag (2908) and $\beta$-actin (4970) antibodies were obtained from Cell Signaling Technology. Cyclin A1 (ab53699) was purchased from Abcam. c-Myc siRNA (sc-29226) and control siRNA (sc-37007) were purchased from Santa Cruz Biotechnology. Other materials were purchased from commercial sources.

\section{Plasmid construction}

The EBP50-shRNA-targeting sequence (5'-CACCGACCAGAAACGCAGCAGCAAACTTCAAGAGAGTTTGCTGCTGCGTTTCTGGTTTTTTTG-3') was subcloned into the pRS shRNA cloning plasmid (Origene, TR20003). A control shRNA oligonucleotide that did not match any known human coding cDNA was used as a control. HA-tagged Beclin-1 (Gene Bank ID: NM_003766.3) was constructed in the pCMV6AC-HA vector (Origene, PS100004) by standard subcloning. Myc-tagged EBP50 (Gene Bank ID: NM_004252.4) was created in the pCMV6-AN-Myc vector (Origene, PS100012) by standard subcloning.

\section{Cell culture and transfection}

MCF-7 breast cancer cell lines were purchased from American Type Culture Collection (ATCC) and maintained as described $^{[2]}$. Transient transfection was performed using Lipofectamine2000 (Invitrogen, 11668019) according to the manufacturer's instructions. To generate MCF-7 cells stably expressing EBP50 shRNA, the control-shRNA and EBP50-shRNA plasmids were both transfected into MCF-7 cells. After $24 \mathrm{~h}$ of transfection, stable transfectants were selected using puromycin, and a single clone was amplified by a dilution cloning technique. To generate MCF-7 cells stably expressing Myctagged EBP50, the pCMV6-AN-Myc vector and Myc-tagged EBP50 plasmids were both transfected into MCF-7 cells. After $24 \mathrm{~h}$ of transfection, stable transfectants were selected using neomycin, and a single clone was amplified by a dilution cloning technique. To generate MCF-7 cells stably expressing Beclin-1, the Beclin-1 lentiviral activation particles (Santa Cruz Biotechnology, sc-400139-LAC) and control lentiviral particles were transfected into MCF-7 cells as described in the manual. To generate MCF-7 cells knockout c-Myc, the c-Myc CRISPR/ Cas9 KO plasmid (Santa Cruz Biotechnology, sc-400001) and control plasmid were transfected into MCF-7 cells as described in the manual.

\section{Tissue microarray slides and immunohistochemistry}

In vivo EBP50 or c-Myc expression was detected by immunohistochemistry (IHC) using tissue microarrays (US Biomax, BC081116c), which contained 110 HCC tissue specimens. IHC was performed according to standard protocols as described previously ${ }^{[12]}$.

\section{Analysis of tumor growth}

The female BALB/C nude mice $(18 \pm 1 \mathrm{~g})$ were obtained from the SPF Biotechology, Co Ltd (Beijing, China). The care and treatment of experimental animals was in accordance with institutional guidelines at the Experimental Animal Center of the Chinese Academy of Medical Sciences. As described previously $^{[13]}$, the MCF-7 cells were injected subcutaneously $\left(10^{7}\right.$ cells/tumor) into the left axilla of nude mice $(n=8$ per group). Mice were weighed, and tumor width $(W)$ and length $(L)$ were measured by vernier calipers every day. Tumor volume was estimated based on formula: $V=0.5 \times L \times W^{2}$. Animals were killed 28 or $35 \mathrm{~d}$ after inoculation of the MCF-7 cells, and tumors were extracted and weighed.

\section{Colony formation assay}

The MCF-7 cells stably expressing EBP50 shRNA or the control-shRNA were seeded in 6-well plates at a density of $2 \times 10^{3}$ cells per well and cultured for $7 \mathrm{~d}$. The plates were washed with PBS and stained with crystal violet. And then the images of each well were scanned, and the individual clone types were identified.

\section{Cell growth assays}

The MCF-7 cells were seeded at a density of $2 \times 10^{4}$ cells per well in 24-well plates and the Sulforhodamine B (SRB) proliferation assay was performed to assess cell proliferation.

\section{Immunoprecipitation and Western blot analysis}

Cells were washed 3 times with phosphate-buffered saline (Invitrogen, 10010023) and then harvested and the cell lysates were extracted in co-immunoprecipitation buffer ${ }^{[2]}$. The total cell lysates (5 mg of protein) were subjected to immunoprecipitation as previously described ${ }^{[2]}$. For the Western blot analysis, co-precipitates or total cell lysates were electrophoresed by SDS-PAGE and transferred to PVDF membranes (Millipore, ISEQ00010). The membranes were immunoblotted with the appropriate antibodies and then developed using a ProteinSimple FluorChem HD2 imaging system (ProteinSimple, CA, USA).

\section{Real-time PCR}

Total RNA was extracted from cells using TRIzol reagent (Invitrogen, 15596026). Total RNA (100 ng) was detected by using a SYBR Green One-Step qRT-PCR Kit (Invitrogen, 11736059) as described in the manual. The following primers were used: $\beta$-actin, $5^{\prime}$-CAACTGGGACGACATGGAGAAAAT-3' and 5' -CCAGAGGCGTACAGGGATAGCAC-3'; 
c-Myc, 5' -AATGAAAAGGCCCCCAAGGTAGTTATCC-3' and 5'-GTCGTTTCCGCAACAAGTCCTCTTC-3'; Cdc25A, 5'-AGATAGCAGTGAACCAGG-3' and 5'-TGCATCGGTTGTCAAGG-3'; Cyclin A1, 5'-GAGAGGGAAACTGCAGCTCG-3' and 5'-AGGAGGATATATCTCTTCAT-3'; Cyclin E1, 5'-ATACAGACCCACAGAGACAG-3' and 5' -TGCCATCCACAGAAATACTT-3'.

\section{EdU assay}

Standard protocols for EdU (5-ethynyl-2'-deoxyuridine) assay to detect the active DNA synthesis were used as described in the manual (Invitrogen, C10340). Images were obtained using a Leica SP2 confocal microscope (Leica Microsystems, Exton, PA, USA) and analyzed with Leica confocal software.

\section{Statistical analysis}

Data are represented as the mean \pm SEM. The statistical analyses were performed using Student's $t$-test to compare 2 groups. The $P$ values less than 0.05 were considered significant. All statistical analyses were performed using SPSS 17.0 software.

\section{Results}

EBP50 knockdown promotes cell proliferation and stimualtes c-Myc signaling in MCF-7 cells

To investigate how EBP50 blocks cell proliferation, the relationship between EBP50 and c-Myc expression was examined in human clinical breast cancer tissues. The results showed that EBP50 expression was lower in breast cancer higher grades of malignancy and the c-Myc expression was positive correlation with malignancy grade (Figure 1A). These data suggested that there is a significant correlation between EBP50 and c-Myc expression in breast cancer tissue. We further found that EBP50 knockdown promoted cell proliferation (Figure 1B and 1C) and induced DNA synthesis (Figure 1D and Supplementary Figure S1). The knockdown EBP50 also increased c-Myc expression (Figure 1E) and promoted c-Myc transfer from the cytoplasm to nucleus (Figure 1F). Moreover, silencing of EBP50 induced mRNA expression of Cdc25A, Cyclin E1 and Cyclin A1 (Figure 1G), all of which are transcription products regulated by c-Myc; the protein expression of these elements was also increased (Figure $1 \mathrm{H}$ ). These data suggest that silencing of EBP50 stimulates c-Myc signaling, which in turn promotes the proliferation of MCF-7 cells.

\section{EBP50 knockdown promotes cell proliferation by stimulating} c-Myc in vivo and in vitro

Because silencing of EBP50 stimulated c-Myc signaling in MCF-7 cells (Figure 1), we further explored whether the silencing EBP50 also induced cell proliferation through this signaling pathway. The results showed that EBP50 silencing increased colony formation and DNA synthesis and promoted cell growth (Figure 1B-1D), whereas knockdown of c-Myc decreased colony formation (Figure 2A and 2B) and DNA synthesis (Figure 2C and 2D) in EBP50-silenced MCF-7 cells. Moreover, silencing c-Myc decreased the tumor growth of
MCF-7 EBP50-silencing cells in nude mice (Figure 2E-2H). These data suggest that EBP50 knockdown promoting cell proliferation depends on c-Myc in vivo and in vitro.

\section{EBP50 promotes c-Myc degradation}

Because silencing of EBP50 induced c-Myc expression and thus promoted the proliferation of breast cancer cells (Figure 1 and 2), we further explored whether EBP50 regulated c-Myc expression by affecting its transcription or degradation. The results showed that c-Myc mRNA expression levels did not differ between EBP50-silenced cells and control cells (Figure 3A), but knockdown of EBP50 increased the expression of c-Myc (Figure 3B). Likewise, overexpression of EBP50 decreased the expression of c-Myc but did not affect levels of c-Myc mRNA (Figure 3C and 3D). Furthermore, knockdown of EBP50 increased c-Myc degradation in cycloheximide (CHX)-treated MCF-7 cells (Figure 3E). Under the same conditions, overexpression of EBP50 promoted c-Myc degradation (Figure 3F). Taken together, these results indicate that EBP50 promotes the degradation of c-Myc.

\section{EBP50 promotes the lysosomal degradation of c-Myc}

As cytosolic proteins are typically degraded by either the ubiquitin proteasome pathway or the autophagy-lysosomal pathway, we next investigated which pathway participates in EBP50-mediated degradation of c-Myc. Treatment with the specific proteasome inhibitor MG132 did not affect c-Myc degradation, whereas blockage of the lysosomal pathway using chloroquine (CQ) significantly attenuated c-Myc degradation in cells overexpressing EBP50 (Figure 4A). Above data suggested that EBP50 induced the lysosomal degradation of c-Myc. We previously reported that EBP50 activates autophagy by stimulating Beclin-1, which triggers the autophagic core complex ${ }^{[2]}$. Hence, we further examined whether EBP50mediated lysosomal degradation of c-Myc was dependent on Beclin-1. Silencing of Beclin-1 delayed this degradation in cells overexpressing EBP50 (Figure 4B), and overexpression of Beclin-1 accelerated the degradation of c-Myc in sh-EBP50 cells (Figure 4C). These data suggest that EBP50 induces the lysosomal degradation of c-Myc by activating Beclin-1.

\section{p62 recognizes c-Myc as a substrate of lysosomal degradation by binding to c-Myc}

p62/SQSTM1 (Sequestosome 1), an autophagic cargo protein, recognizes the substrates of lysosomal degradation by binding to them. Hence, we next investigated whether p62 binds to c-Myc. Co-immunoprecipitation and Glutathione S-transferase (GST) pull-down assays showed that p62 could bind to c-Myc both in vivo and in vitro (Figure 5A and 5B). Additionally, silencing of EBP50 decreased the interaction between p62 and c-Myc (Figure 5C). Interestingly, overexpression of EBP50 also reduced the binding of p62 and c-Myc, and inhibiting autophagy using CQ promoted the interaction between p62 and c-Myc (Figure 5D). Furthermore, EBP50 promoted the integration of c-Myc and p62 when the lysosomal pathway was inhibited by CQ (Figure 5E). These data suggest that p62 
A
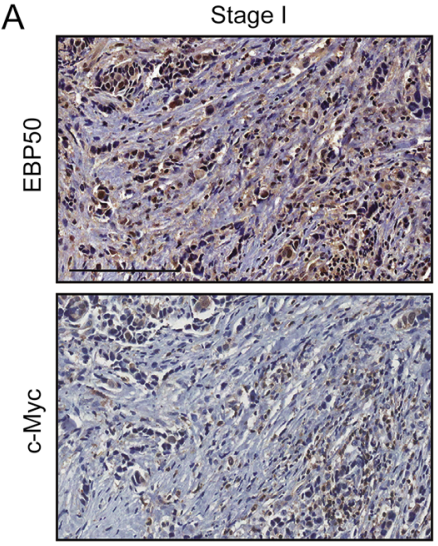
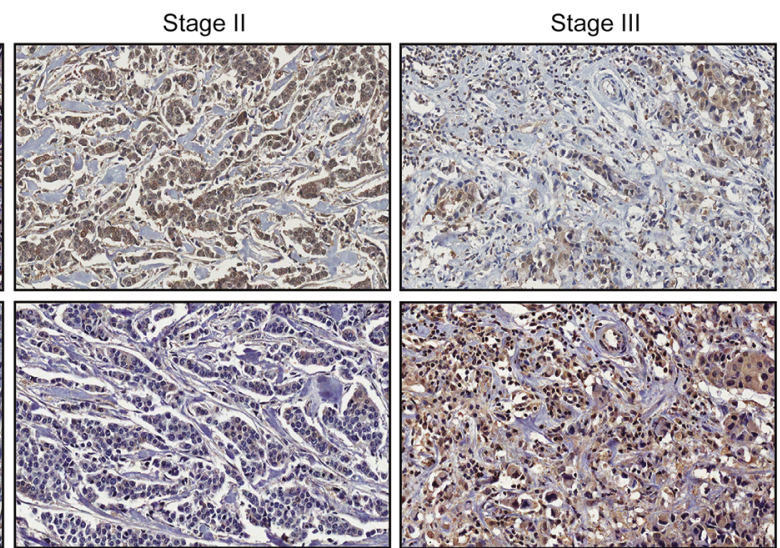

B
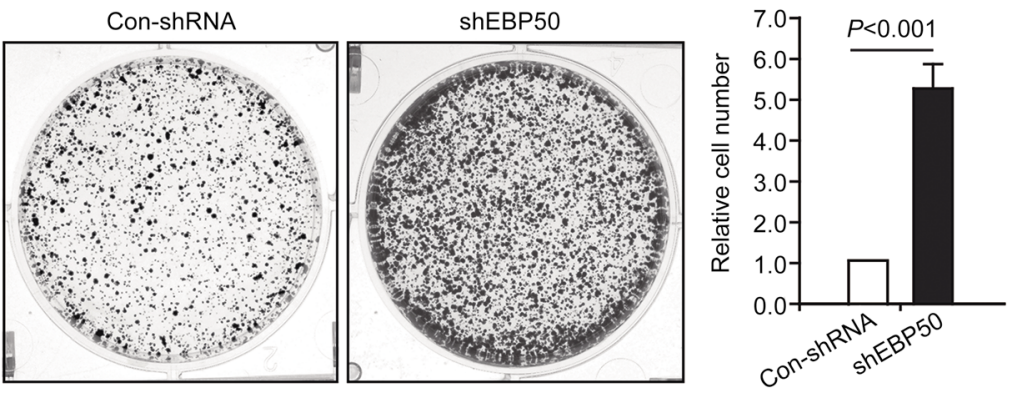

D
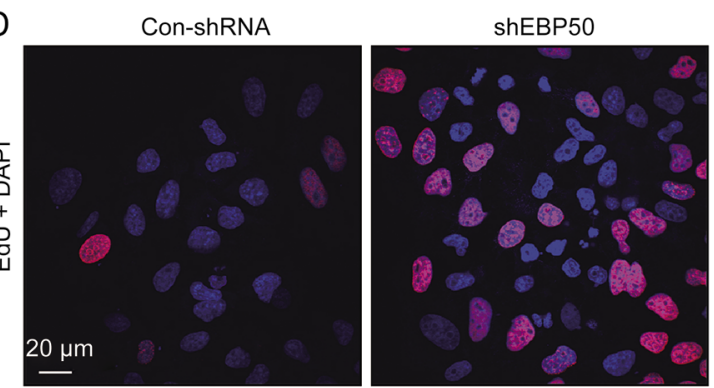

E

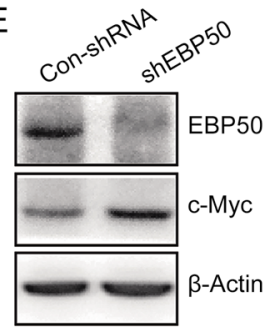

C

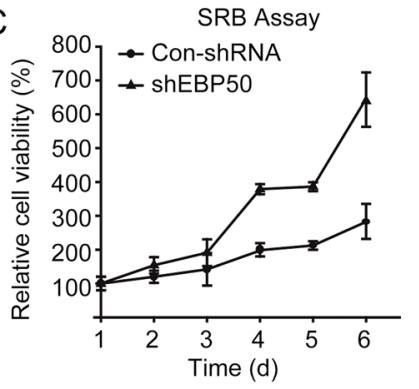

F

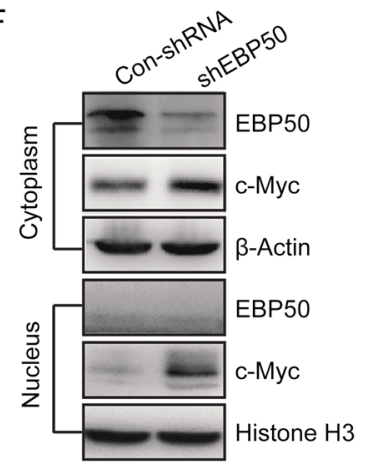

G

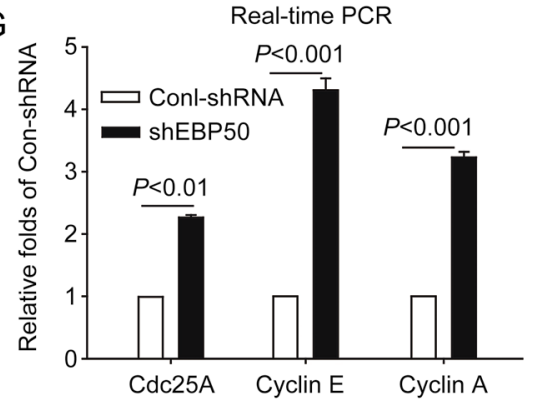

$\mathrm{H}$

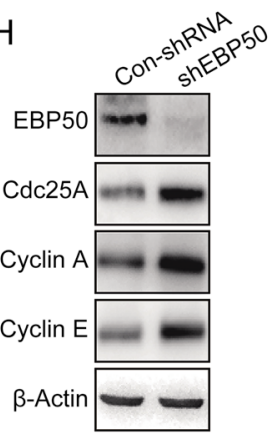

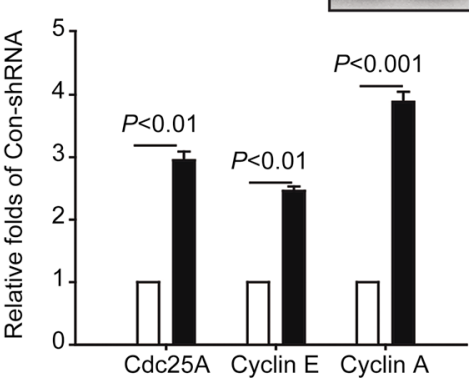

Figure 1. Knockdown of EBP50 promotes cell proliferation and stimulates c-Myc signaling in MCF-7 breast cancer cells. (A) The expression of EBP50 and C-Myc was correlation with malignancy grade in breast cancer tissues. The expression of EBP50 and c-Myc was stained by IHC as indicated in Materials and methods. Scale bar, $200 \mu \mathrm{m}$. (B, C) Knockdown of EBP50 promoted the proliferation of MCF-7 cells. Colonies were counted (B) and proliferating cells were measured using SRB assays (C) after MCF-7 cells expressing either control shRNA or EBP50 shRNA were cultured for 7 d. (D) Knockdown of EBP50 increased DNA synthesis in MCF-7 cells. Data are representative images of Edu labelling as indicated in the Materials and methods. (E) Knockdown of EBP50 increased the expression of c-Myc in MCF-7 cells. MCF-7 cells expressing either control shRNA or EBP50 shRNA were cultured and the cell lysates were detected by Western blotting using c-Myc antibodies. (F) Knockdown of EBP50 increased the nuclear import of c-Myc. Subcellular fractions were extracted, and c-Myc expression was detected by Western blotting. (G) Silencing of EBP50 increased the levels of RNA transcription products of c-Myc. Total RNA was isolated from MCF-7 cells expressing either control shRNA or EBP50 shRNA, and the mRNA levels of Cdc25A, Cyclin A1 and Cyclin E1 were analyzed by fluorescent quantitative RT-PCR as indicated in the Materials and methods. (H) Silencing of EBP50 increased the expression of transcription products of c-Myc. Cell lysates were detected by Western blotting using Cdc25A, Cyclin A1 and Cyclin E1 antibodies. Data are presented as the mean $\pm \operatorname{SEM}(n=4)$. 
A

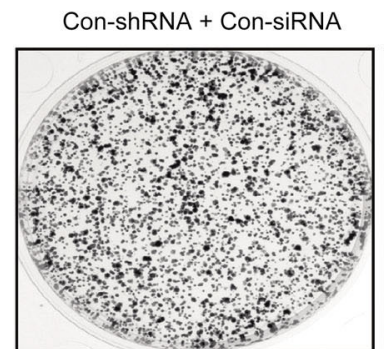

Con-shRNA + si-c-Myc

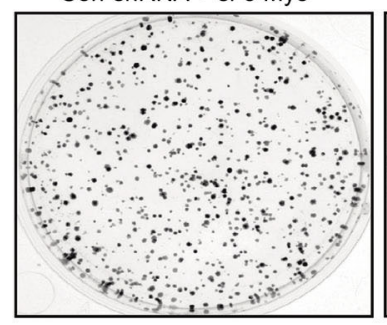

C

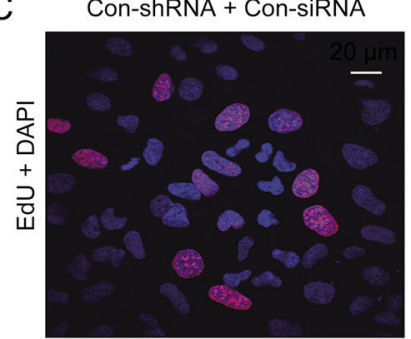

Con-shRNA + si-c-Myc

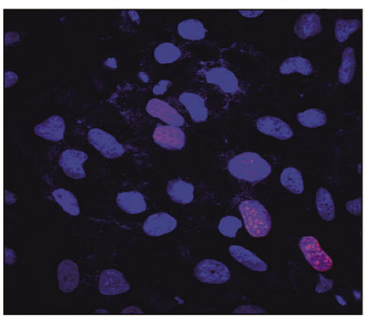

shEBP50 + Con-siRNA

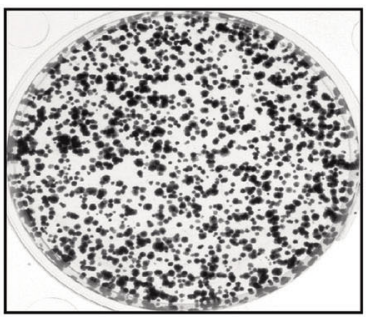

shEBP50 + si-c-Myc

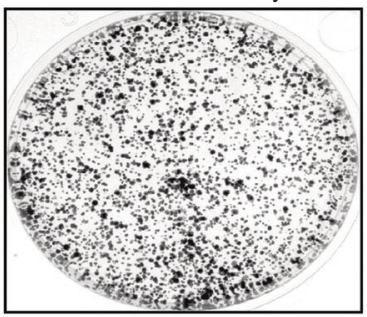

shEBP50 + Con-siRNA

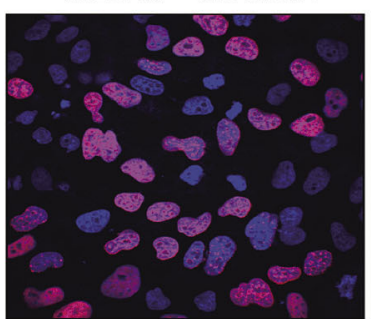

shEBP50 + si-c-Myc

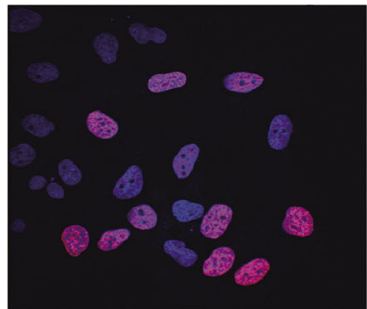

B

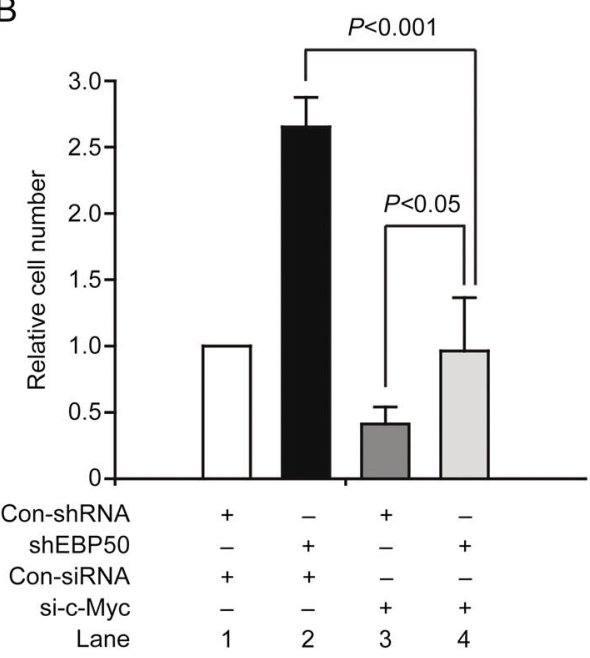

D

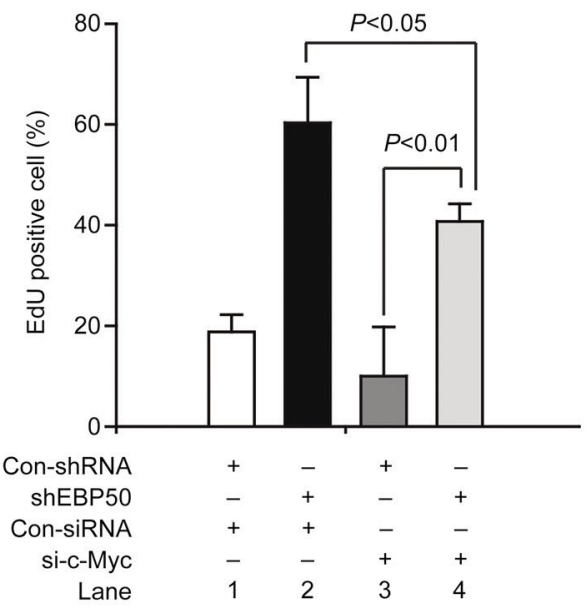

Figure 2A-2D. EBP50 stimulates C-Myc in MCF-7 breast cancer cells to promote their proliferation. (A, B) Silencing of c-Myc eliminates the tumor proliferation induced by EBP50 knockdown in MCF-7 cells. Colonies were counted after MCF-7 cells were transfected with Control-siRNA or si-c-Myc and then cultured for $7 \mathrm{~d}$. (C, D) Silencing of c-Myc inhibits DNA synthesis in EBP50-knockdown cells. After MCF-7 cells were transfected with Control-siRNA or si-c-Myc for $24 \mathrm{~h}$, DNA synthesis was assessed using Edu staining as indicated in the Materials and methods. Data are presented as the mean \pm SEM $(n=4)$. Continued on the next page.

recognizes c-Myc as a substrate of lysosomal degradation by binding to c-Myc during EBP50-induced lysosomal degradation of c-Myc.

EBP50 suppresses cell proliferation by stimulating Beclin-1mediated lysosomal degradation of c-Myc in MCF-7 breast cancer cells

EBP50 promotes the lysosomal degradation of c-Myc by acting through Beclin-1 (Figure 4B and 4C). Thus, we next explored whether EBP50 suppressed the proliferation of breast cancer cells following the stimulation of Beclin-1 expression. Overexpression of Beclin-1 suppressed colony formation (Figure 6A and 6C) and DNA synthesis (Figure 6B and 6D) in EBP50-knockdown cells, which suggests that EBP50- mediated suppression of MCF-7 cell proliferation depends on increased levels of Beclin-1. Furthermore, overexpression of Beclin-1 reduced the nuclear import of c-Myc (Figure 6E) and decreased the expression of transcription products regulated by c-Myc (Figure 6F and 6G), which suggests that stimulation of autophagy by the overexpression of Beclin- 1 inhibits c-Mycrelated signaling. Together, these data suggest that EBP50 suppresses cell proliferation by stimulating Beclin-1-mediated lysosomal degradation of c-Myc in MCF-7 breast cancer cells.

EBP50 suppresses cell proliferation by effecting Beclin-1/p62/ c-Myc related signaling pathway in vivo

We next explored effect of EBP50/Beclin-1/p62/c-Myc signaling on cell proliferation in vivo. We found that silencing 
E

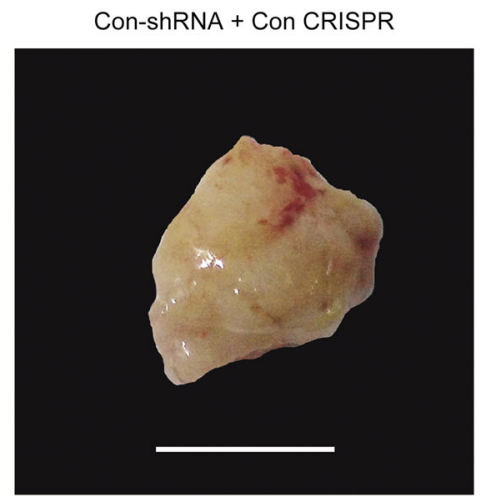

$\mathrm{F}$

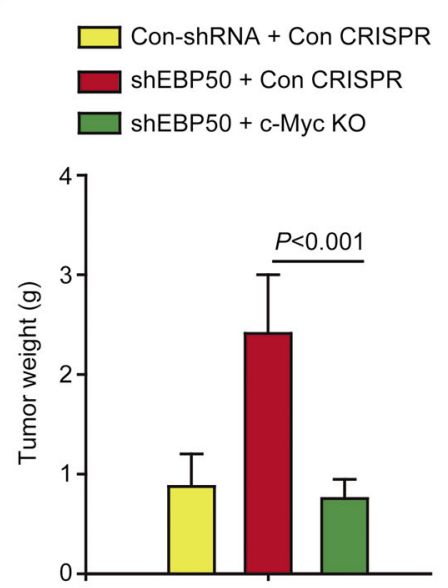

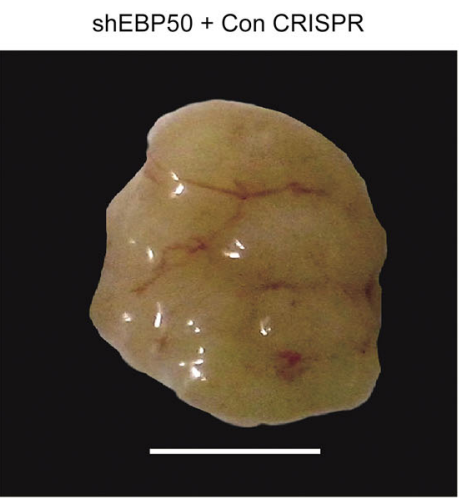

G $\rightarrow$ Con-shRNA + Con CRISPR

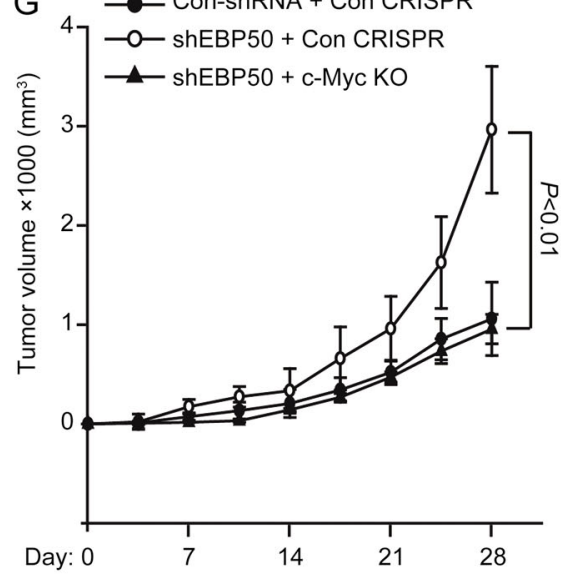

shEBP50 + c-Myc KO

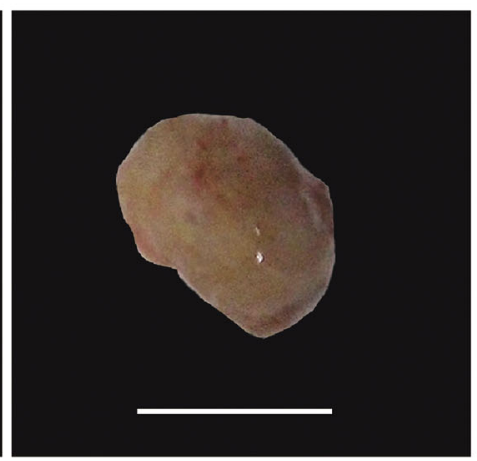

$\mathrm{H}$

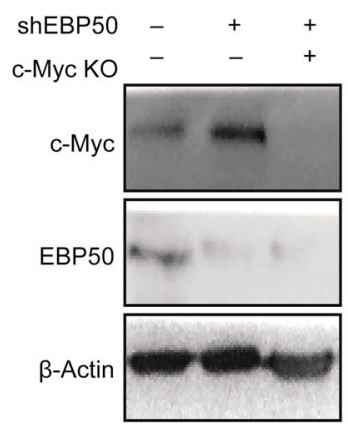

Figure 2E-2H. EBP50 stimulates c-Myc in MCF-7 breast cancer cells to promote their proliferation. (E-G) Silencing EBP50 promotes cell proliferation by stimulating C-Myc in nude mice. MCF-7 cells were injected subcutaneously the left axilla of nude mice as indicated in the Materials and methods. Scale bar, $1.0 \mathrm{~cm}$. Data are presented as the mean \pm SEM $(n=8)$. (H) The expression of EBP50 and c-Myc in the tumor tissues. The tumor tissues were homogenized and the expression of EBP50 or c-Myc was detected by Western blot.

EBP50 induced the tumor growth and overexpressing Beclin-1 reduced it in tumor-bearing mice with EBP50-silencing MCF-7 cells (Figure 7A-7C), suggesting that silencing EBP50 promotes cell proliferation by inhibiting Beclin-1. Moreover, overexpression of Beclin-1 decreased the expression of c-Myc and p62 in tumor-bearing mice with EBP50-silencing MCF-7 cells (Figure 7D). Together, these data suggested that EBP50 suppresses tumor proliferation by regulating Beclin-1/p62/c-Myc signal pathway (Figure 7E).

\section{Discussion}

Cytoplasmic EBP50 suppresses cell proliferation by regulating various signaling pathways in breast cancer cells. Overexpressing EBP50 decreased the tumor growth and silencing EBP50 increased it in MCF-7 cells (Figure 1 and Supplementary Figure S2). In MDA-MB-231 cells, EBP50 suppresses cell growth by promoting apoptosis ${ }^{[14]}$. EBP50 also inhibits epidermal growth factor (EGF)-induced breast cancer cell proliferation by blocking extracellular signal-regulated kinase 1 and 2 (ERK1/2) and Akt phosphorylation ${ }^{[3]}$. In our current study, we found that EBP50 inhibits the cell proliferation by suppressing c-Myc signaling in MCF-7 cells (Figure 2). Our report suggests that EBP50 plays a significant role in suppressing c-Myc signaling to trigger the inhibition of tumor proliferation in MCF-7 cells.

In the development and progression of breast cancer, c-Myc transfers from cytoplasm to nucleus and later promotes the transcription progression on regulators of cell cycle, such as cyclin A1, cyclin E1, and Cdc25A, to drive cell proliferation ${ }^{[6,7]}$. Hence, the nuclear c-Myc, not the cytoplasmic c-Myc, plays a pivotal role on cell proliferation. In our current studies, we observed that silencing EBP50 induced the expression of c-Myc in the whole cell lysates (Figure 1E). And we further found that silencing EBP50 increased the level of nuclear c-Myc (Figure 1F) and the expressions of downstream molecules (such as cyclin A1, cyclin E1 and Cdc25A) (Figure 1H), suggesting that silencing EBP50 stimulated the c-Myc signaling pathway. In previous studies, we found that overexpressing EBP50 stimulates autophagy and silencing EBP50 restrains it in cytoplasm ${ }^{[2]}$. In our current studies, we observed that silencing EBP50 delayed the autophagic lysosomal degradation of c-Myc (Figure 4), not affect the RNA level of c-Myc (Figure $3 \mathrm{~A}$ and $\mathrm{3C}$ ). Together, these above observations suggested that silencing EBP50 blocked the autophagic lysosomal 
A
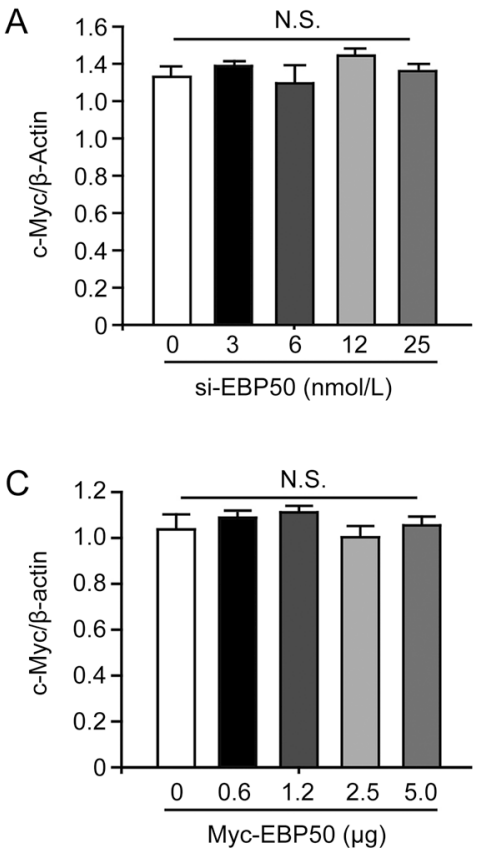

E

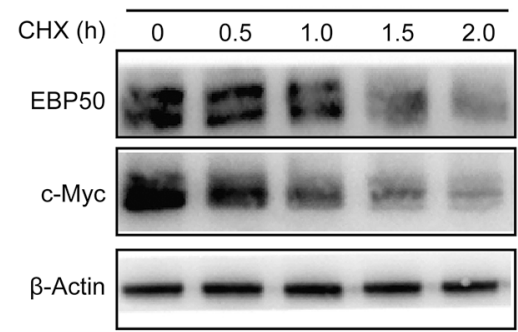

D
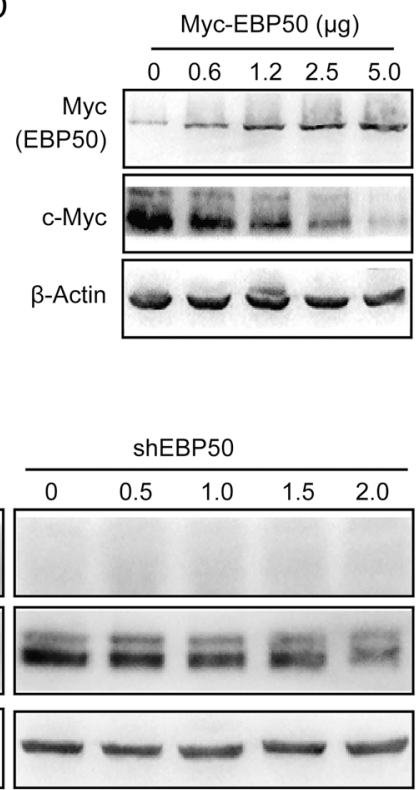

$\mathrm{F}$

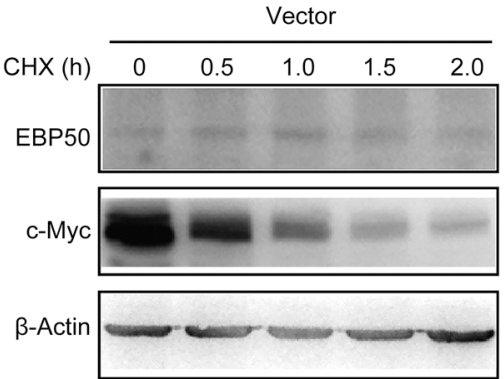

B
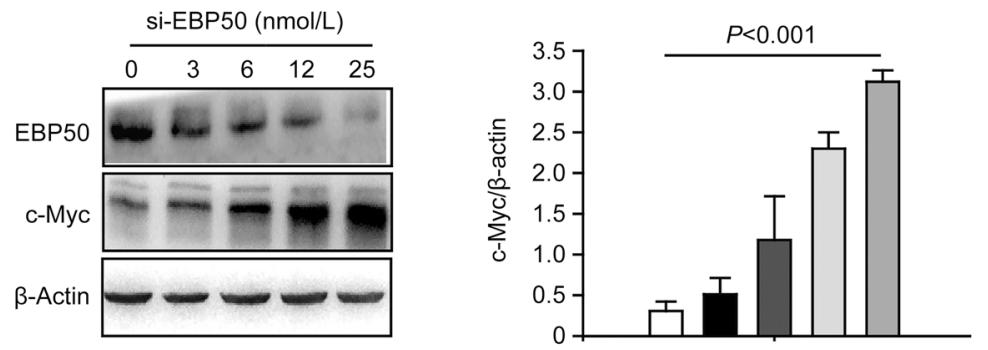
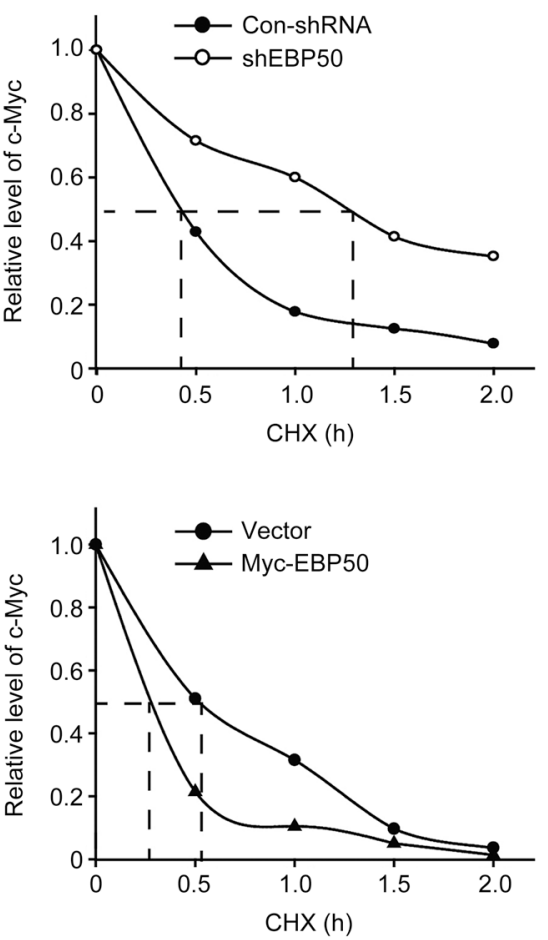

Figure 3. EBP50 promotes the degradation of c-Myc. (A) Silencing of EBP50 did not affect the mRNA expression of c-Myc in MCF-7 cells. Cells were transfected with the indicated concentrations of si-EBP50, and total RNA was isolated. C-Myc mRNA expression was analyzed by fluorescent quantitative RT-PCR as indicated in the Materials and methods. (B) Knockdown of EBP50 increased the expression of c-Myc in MCF-7 cells. MCF-7 cells were transfected with the indicated concentrations of si-EBP50. Protein lysates were analyzed by immunoblotting with the indicated antibodies. (C) Up-regulation of EBP50 did not affect the mRNA expression of c-Myc in MCF-7 cells. Cells were transfected with the indicated concentrations of Myc-EBP50, and total RNA was isolated. C-Myc mRNA expression was analyzed by fluorescent quantitative RT-PCR as indicated in the Materials and methods. (D) Overexpression of EBP50 decreases the expression of c-Myc in MCF-7 cells. MCF-7 cells were transfected with the indicated concentrations of Myc-EBP50. Protein lysates were analyzed by immunoblotting with the indicated antibodies. (E) Knockdown of EBP50 inhibits the degradation of C-Myc in MCF-7 cells. Cells were transfected with Flag-c-Myc plasmids for $24 \mathrm{~h}$ and treated with $\mathrm{CHX}(20 \mu \mathrm{mol} / \mathrm{L})$ for the indicated times, and then cell lysates were detected by Western blotting with an anti-Flag antibody. (F) EBP50 induces the degradation of C-Myc in MCF-7 cells. Cells were cotransfected with Myc-EBP50 and Flag-c-Myc for $24 \mathrm{~h}$ and treated with CHX $(20 \mu \mathrm{mol} / \mathrm{L})$ for the indicated times, and then cell lysates were detected by Western blotting with an anti-Flag antibody. Data are presented as the mean \pm SEM $(n=4)$. NS, non-significant; $\mathrm{CHX}$, cycloheximide. 
A

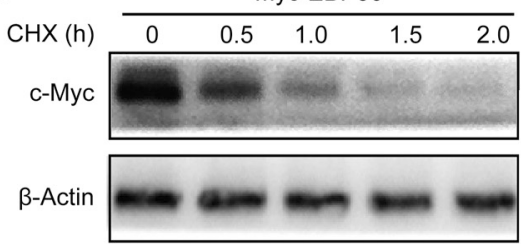

B
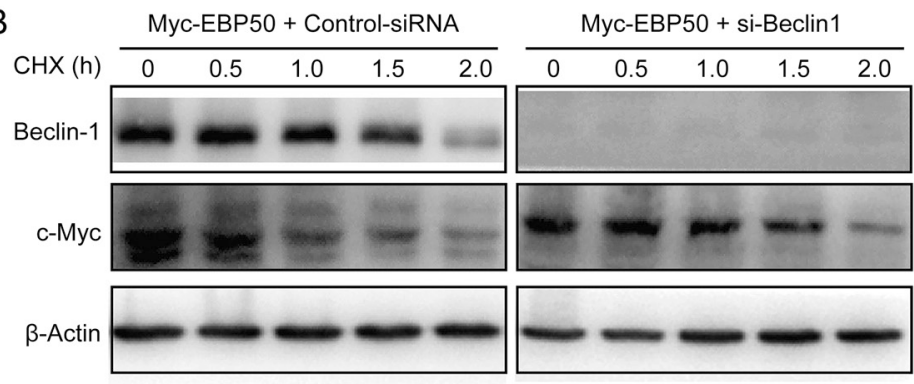

$\mathrm{C}$
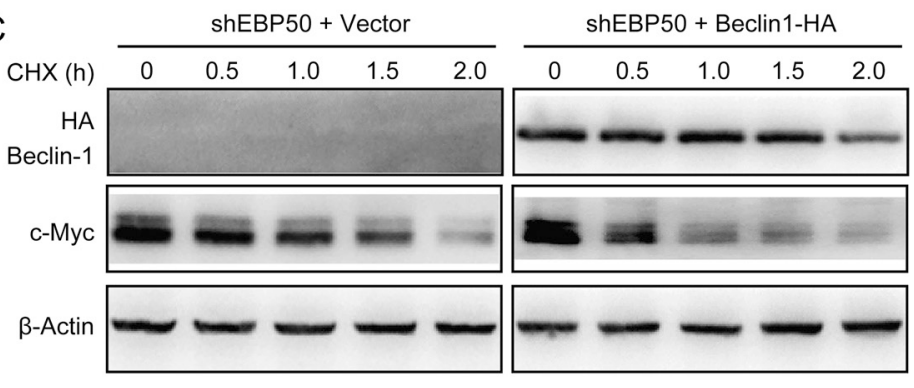
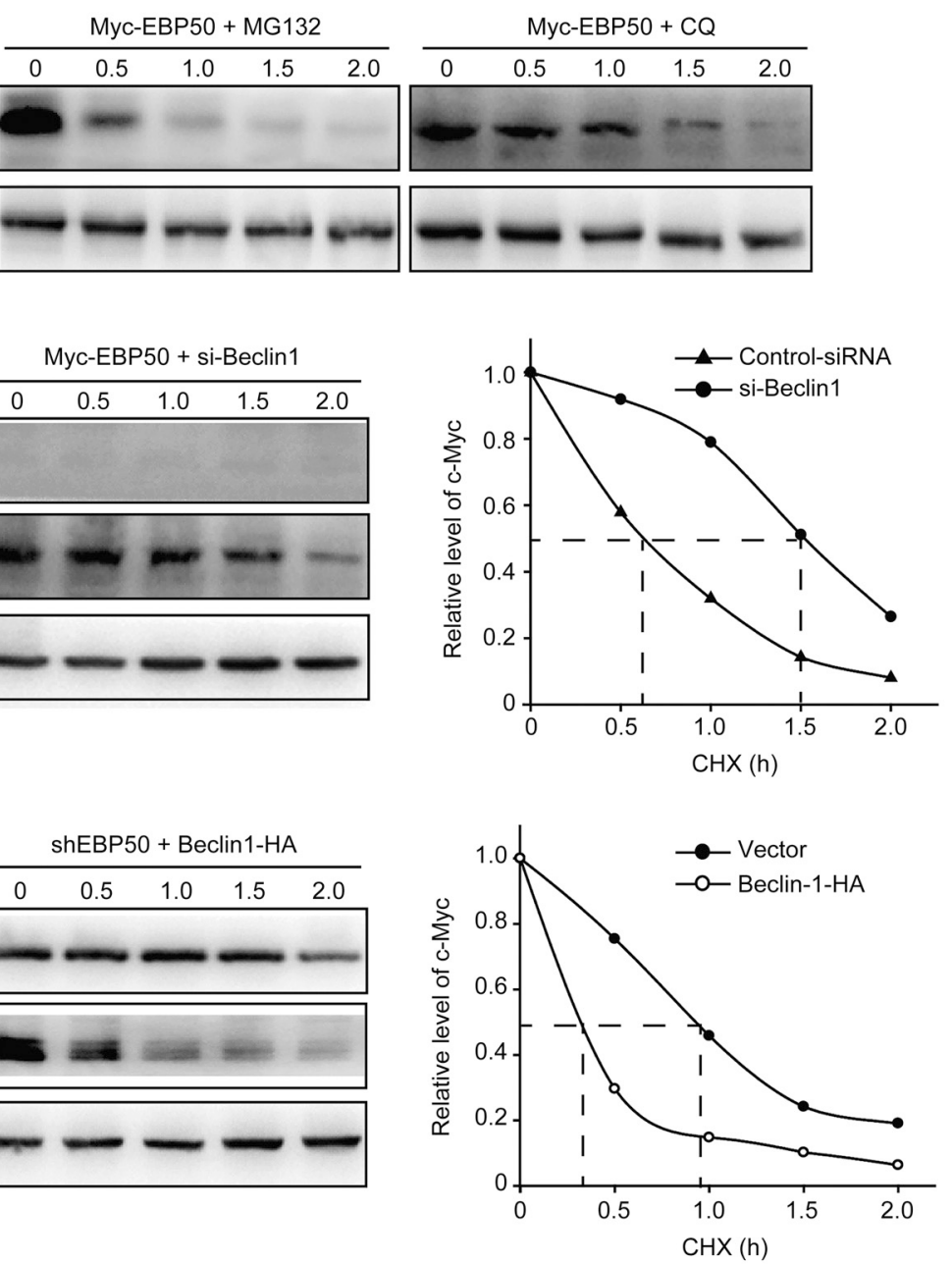

Figure 4. EBP50 promotes C-Myc degradation through the autophagic lysosomal pathway. (A) CQ inhibition of the lysosomal pathway blocks the degradation of c-Myc induced by EBP50. MCF-7 cells were transfected with Flag-c-Myc vector for $24 \mathrm{~h}$ and then treated with MG132 (2.5 $\mu$ mol/L) for $2 \mathrm{~h}$ or $\mathrm{CQ}(50 \mu \mathrm{mol} / \mathrm{L})$ for $6 \mathrm{~h}$ before the addition of $\mathrm{CHX}(20 \mu \mathrm{mol} / \mathrm{L})$ for the indicated times. C-Myc (Flag) expression was detected by immunoblotting with the indicated antibodies. (B) Inhibition of autophagy by silencing Beclin-1 promotes the degradation of C-Myc. MCF-7 cells were cotransfected

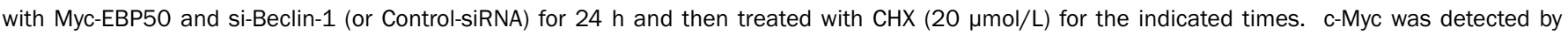
immunoblotting with the indicated antibodies. (C) Activation of autophagy by overexpression of Beclin-1 accelerates the degradation of C-Myc. MCF-7 cells were cotransfected with Flag-c-Myc and Beclin-1-HA vector for $24 \mathrm{~h}$ and then treated with $\mathrm{CHX}(20 \mu \mathrm{mol} / \mathrm{L})$ for the indicated times. C-Myc (Flag) was then detected by immunoblotting with the indicated antibodies. $\mathrm{CHX}$, cycloheximide; $\mathrm{CQ}$, chloroquine.

degradation of c-Myc and increased the expression of c-Myc in cytoplasm in order to transfer c-Myc from cytoplasm to nucleus and then promote the transcription of c-Myc downstream molecules.

c-Myc degradation has been been shown to restrain cancer proliferation and various factors inhibit tumor growth by causing c-Myc degradation. Pim kinase, an oncogene important in leukemia, lymphoma, prostate and pancreatic cancer, stabilizes c-Myc and inhibits its degradation ${ }^{[15]}$. TM, a potent SIRT2-specific inhibitor, exhibits broad anticancer activity by promoting c-Myc ubiquitination and degradation ${ }^{[16]}$. Gamabufotalin inhibits cell growth and induces apoptosis by evoking the ubiquitination and degradation of c-Myc in multiple myeloma cells ${ }^{[17]}$. The above studies suggest that stimulation of c-Myc degradation is effective for inhibiting tumor cell pro- liferation. However, the above reports focused only on ubiquitin-mediated degradation of c-Myc. In the present study, we showed that EBP50, an activator of autophagy, causes lysosomal degradation of c-Myc to suppress the growth of MCF-7 breast cancer cells (Figure 4 and 6). Overall, the above findings suggest that c-Myc is degraded through both the lysosomal and ubiquitin pathway and that promoting c-Myc degradation inhibits tumor cell proliferation.

p62, an autophagic cargo protein, binds to endogenous autophagy substrates during autophagic lysosomal degradation. GATA4 acts as an activator of hepatoblastoma cell proliferation ${ }^{[18]}$; after binding to p62, GATA4 is degraded through the autophagic lysosomal pathway ${ }^{[19]}$. Our results showed that p62 binds to c-Myc, causing c-Myc degradation through the lysosomal pathway (Figure 5), which is consistent with 
A

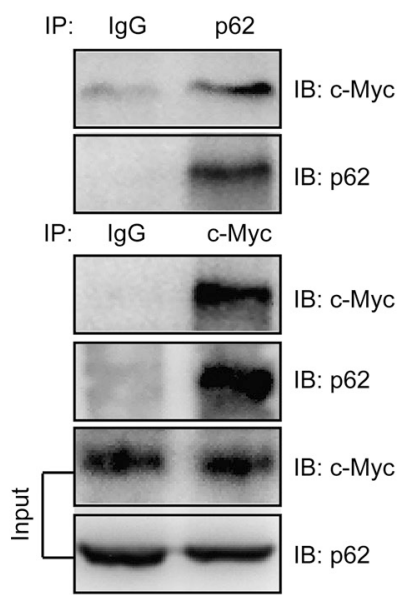

B

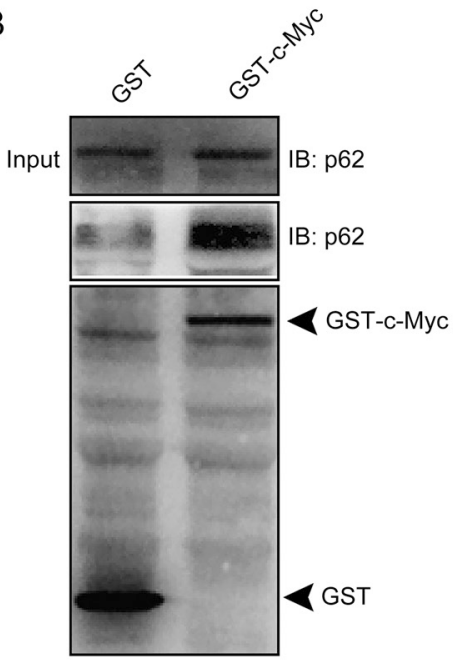

C
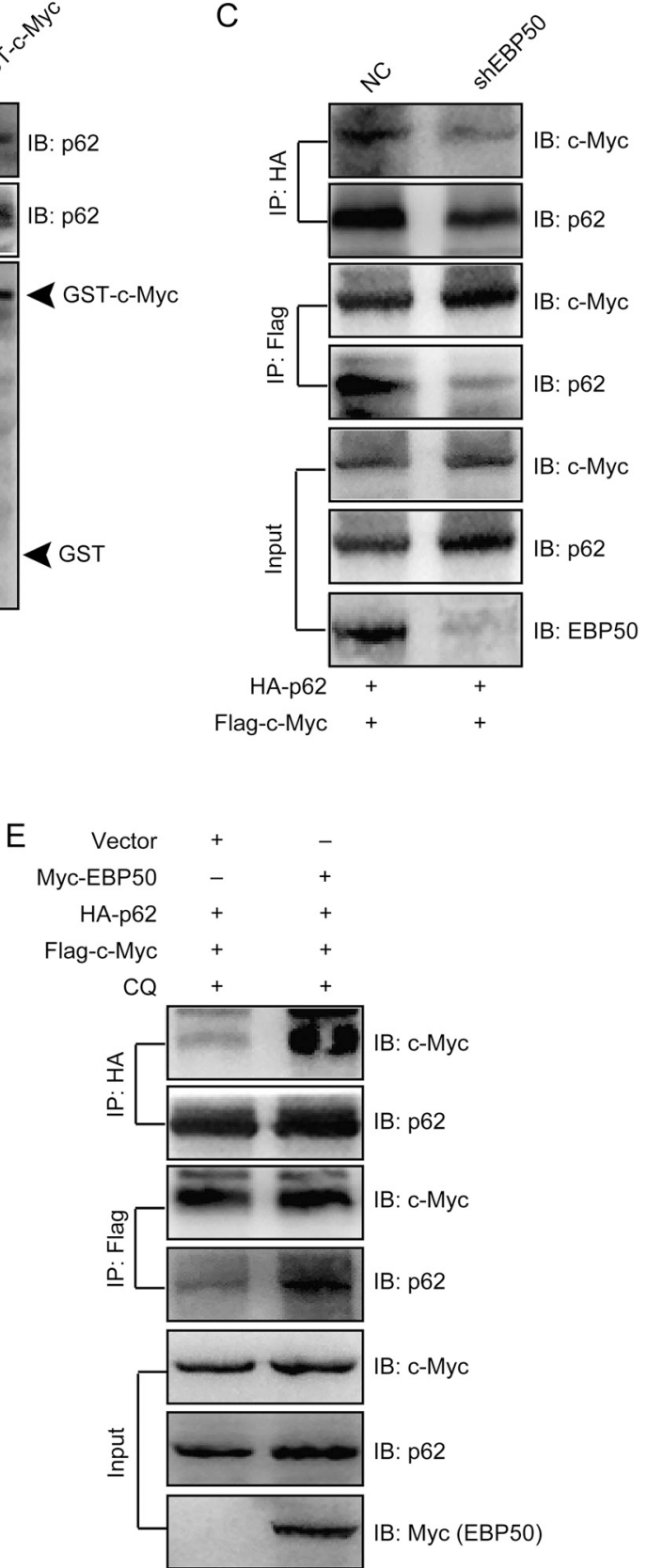

Figure 5. p62 interacts with c-Myc to degrade c-Myc. (A) p62 interacts with c-Myc in MCF-7 cells. Whole-cell lysates were immunoprecipitated with an anti-p62 antibody and blotted with an anti-c-Myc antibody. (B) In vitro binding between p62 and c-Myc. Cell extracts from MCF-7 cells were incubated with equal amounts of GST or GST-c-Myc and analyzed by Western blotting using an anti-p62 antibody. The presence of GST fusion proteins was confirmed by Western blotting using an anti-GST antibody. (C) Silencing of EBP50 reduces the interaction of p62 and c-Myc. Flag-c-Myc was cotransfected with HA-p62 into MCF-7 cells. Whole-cell lysates were immunoprecipitated with an anti-HA antibody and blotted with an anti-c-Myc antibody. (D) Inhibition of autophagy promotes the interaction of p62 and c-Myc. Myc-EBP50 was co-transfected with HA-p62 and Flag-c-Myc into MCF-7 cells for $24 \mathrm{~h}$, and then the cells were treated with CQ $(50 \mu \mathrm{mol} / \mathrm{L})$ for another $6 \mathrm{~h}$. Whole-cell lysates were immunoprecipitated with an anti-HA antibody and blotted with an anti-c-Myc antibody. (E) EBP50 promotes the binding of p62 and c-Myc. Myc-EBP50 was co-transfected with HA-p62 and Flag-c-Myc into MCF-7 cells for $24 \mathrm{~h}$ and then the cells were treated with CQ $(50 \mu \mathrm{mol} / \mathrm{L})$ for another $6 \mathrm{~h}$. Whole cell lysates were immunoprecipitated with an anti-HA antibody and blotted with an anti-c-Myc antibody.

the above report. Moreover, other autophagy-related proteins also regulate the degradation of c-Myc. Valentina et al found that the pro-autophagic protein AMBRA1 regulates cell proliferation by facilitating the degradation of $\mathrm{c}-\mathrm{Myc}^{[20]}$, but they did not discuss AMBRA1 causes the degradation of c-Myc by ubiquitin proteasome pathway or the autophagy-lysosomal pathway. Pietri et al reported CIP2A, an inhibitor of autophagy by stimulating mammalian target of rapamycin complex 1 
A

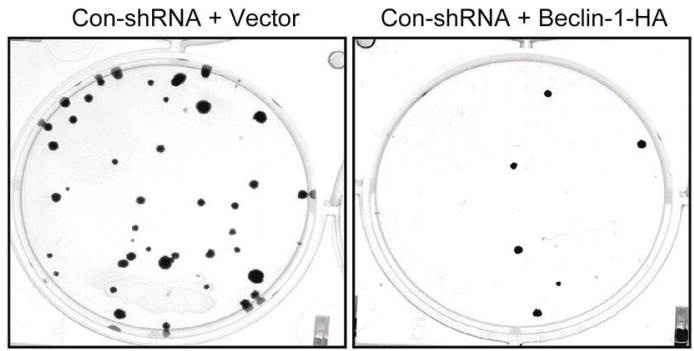

shEBP50 + Vector

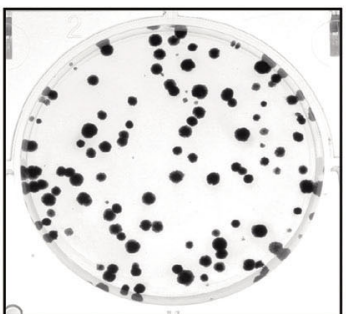

shEBP50 + Beclin-1-HA
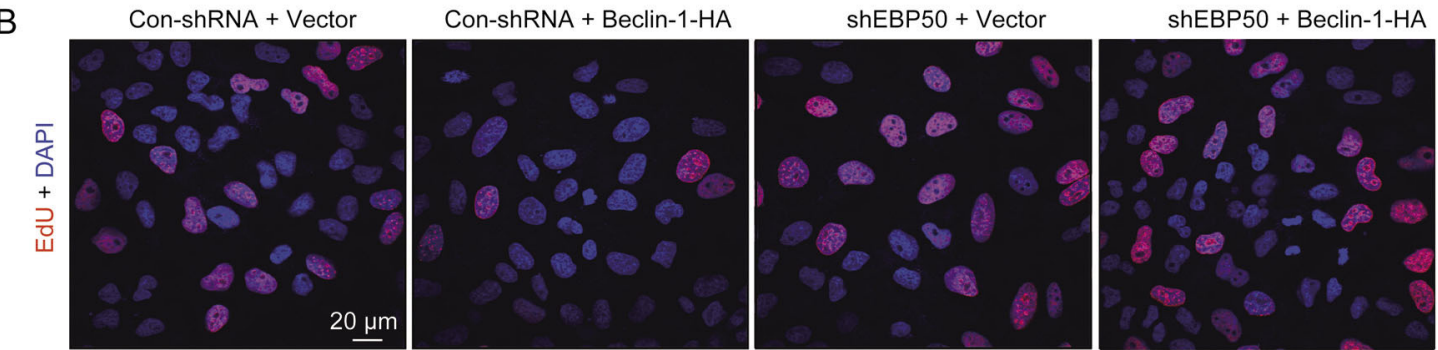

C

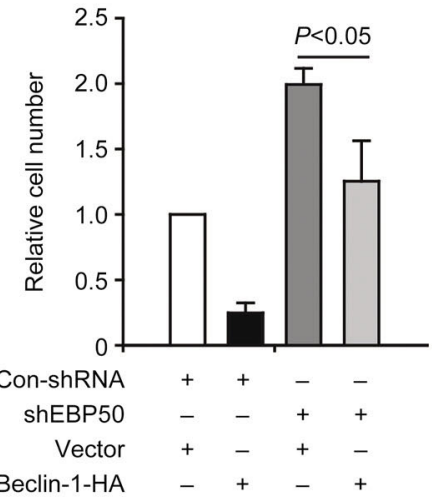

F

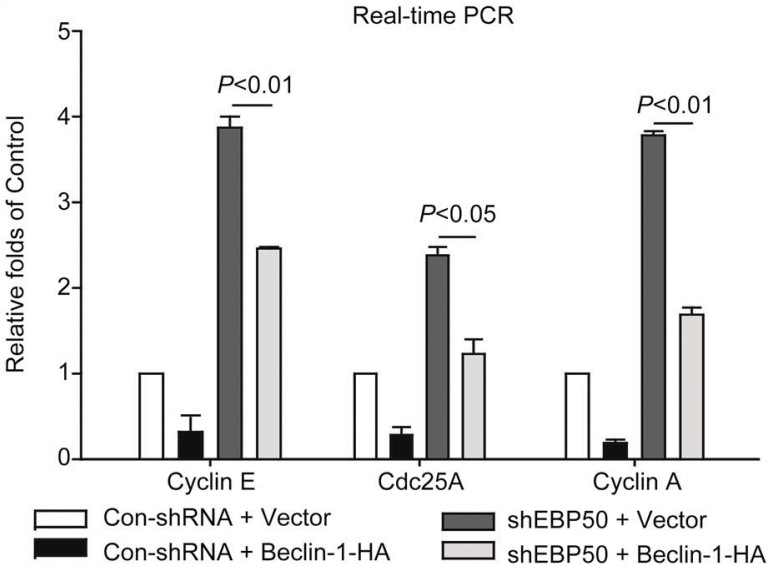

D

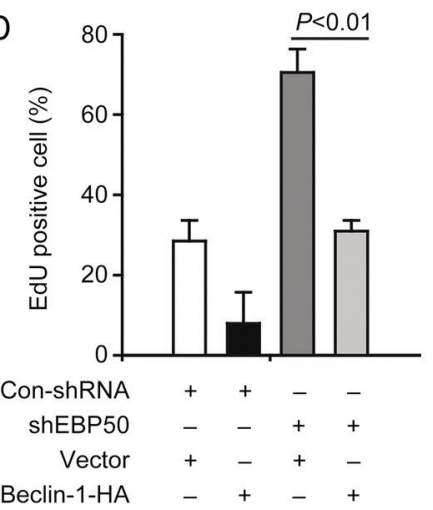

E shEBP50

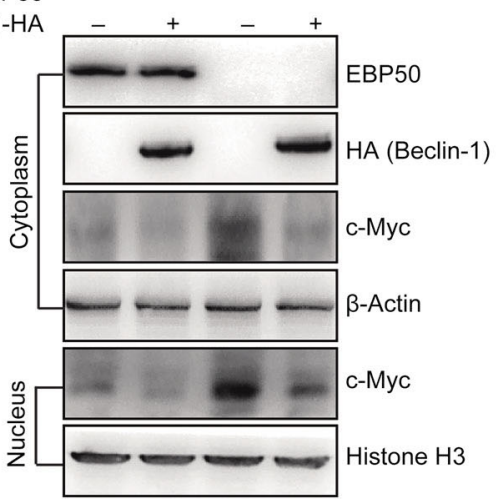

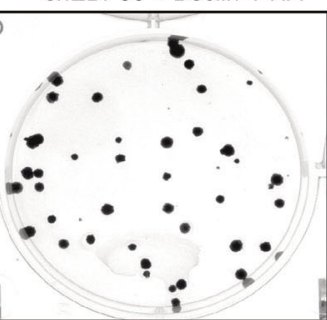

\section{G}

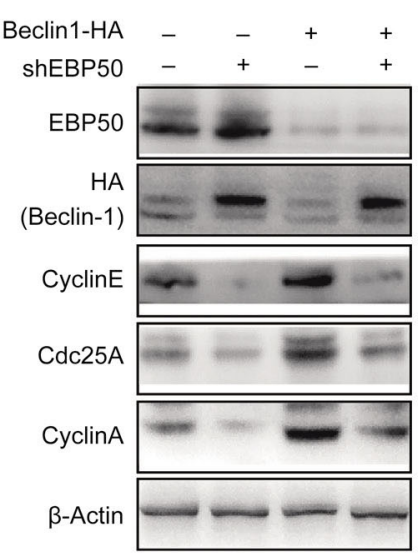

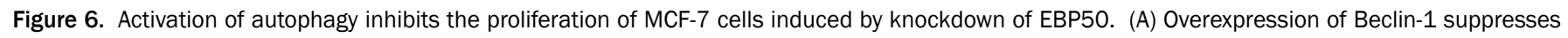

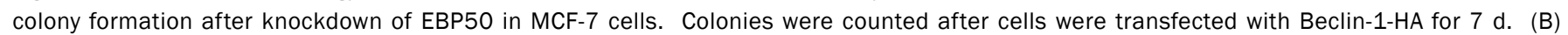

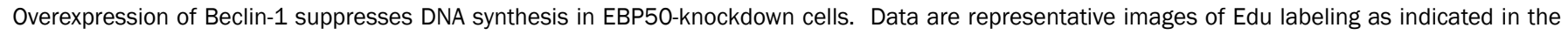

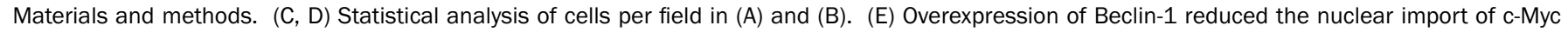

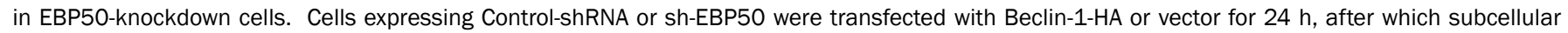

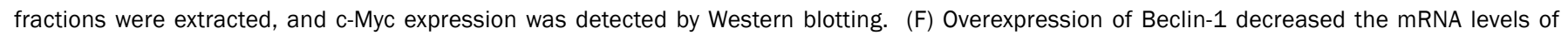

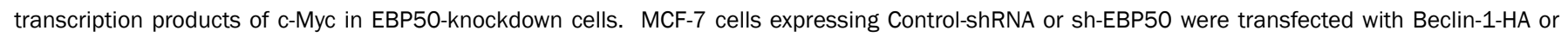

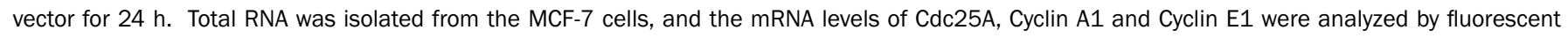

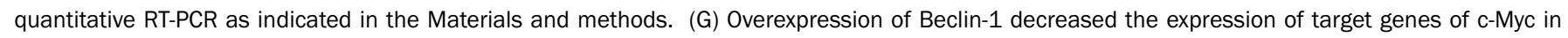

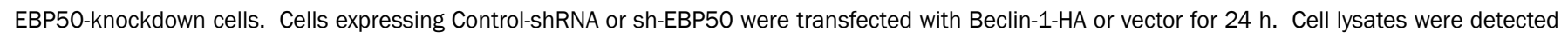
by Western blotting with Cdc25A, Cyclin A1 and Cyclin E1 antibodies. Data are presented as the mean \pm SEM ( $n=4)$. 
A

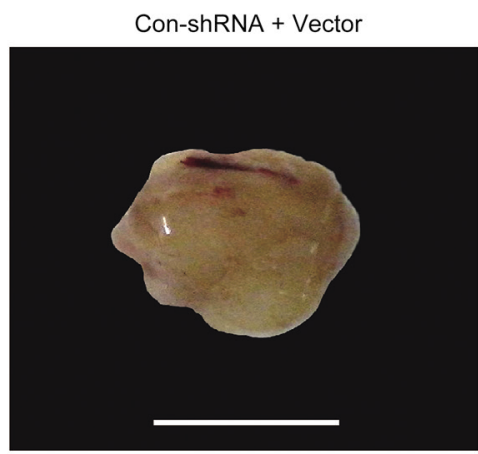

B
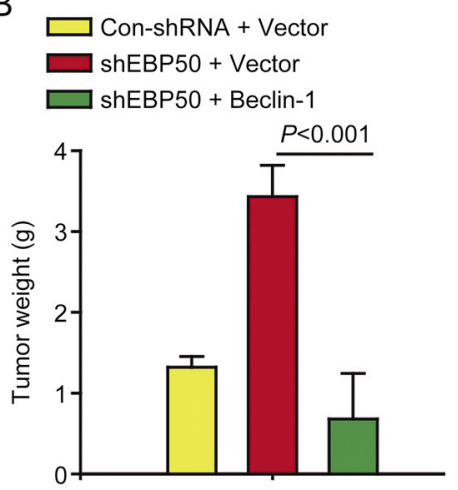

E

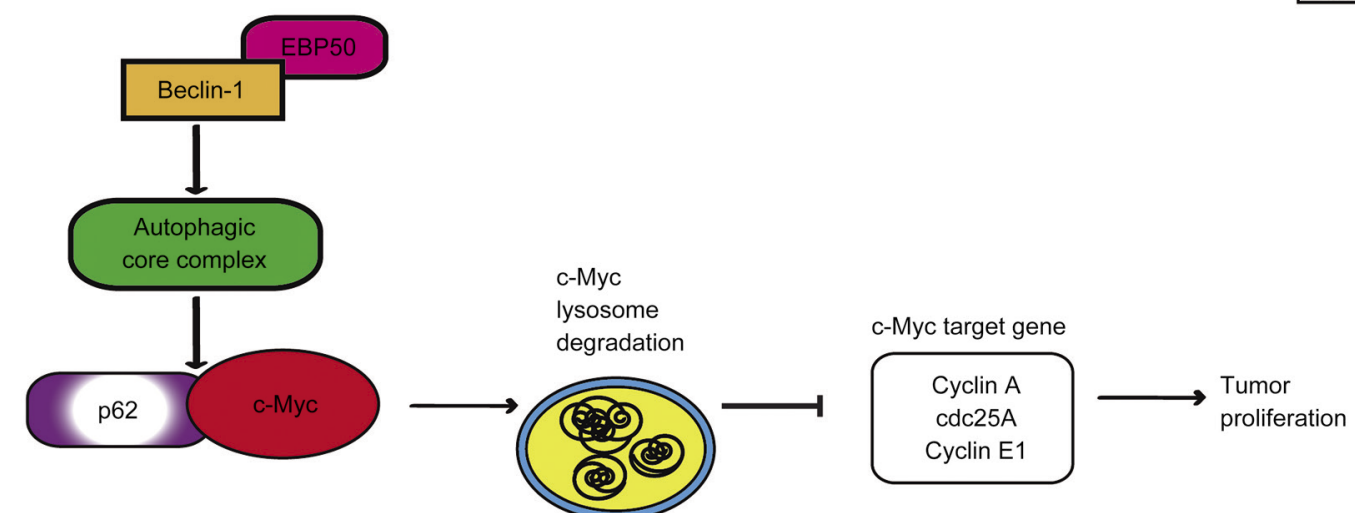

C
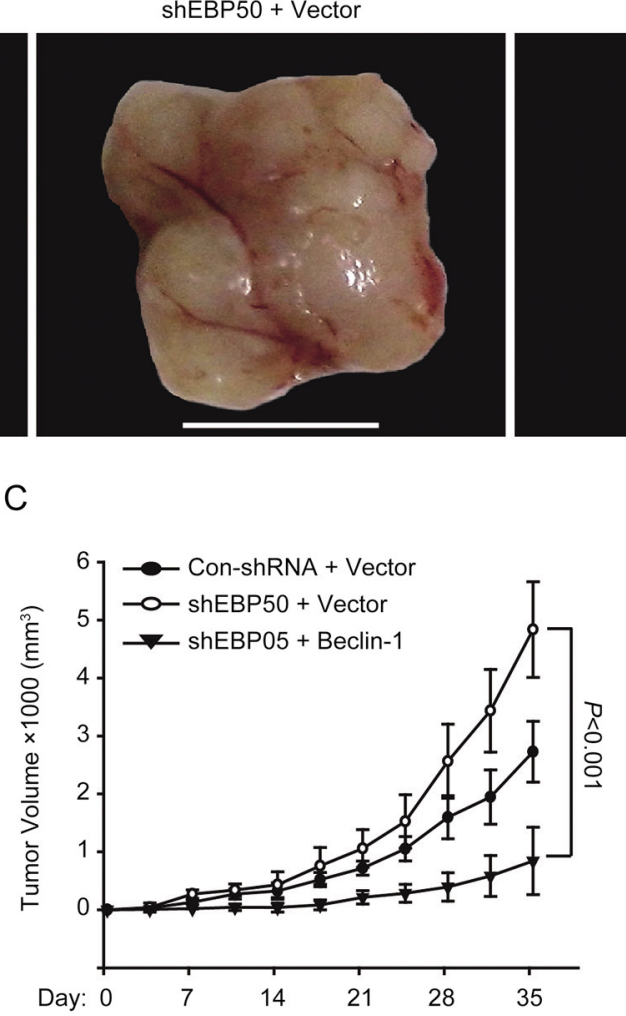

shEBP50 + Beclin-1

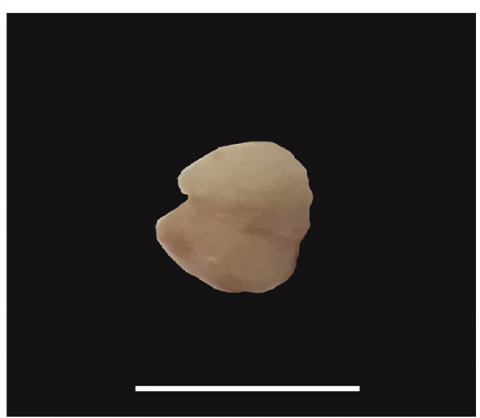

D

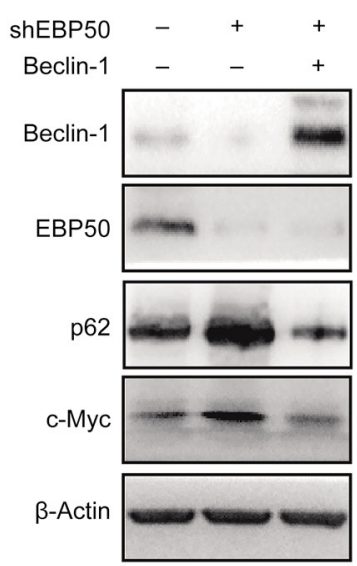

Figure 7. EBP50 suppresses cell proliferation by effecting Beclin-1/p62/c-Myc related signaling pathway in vivo. (A-C) EBP50 suppresses cell proliferation by stimulating Beclin-1 in nude mice. MCF-7 cells were injected subcutaneously the left axilla of nude mice as indicated in the Materials and methods. Scale bar, $1.0 \mathrm{~cm}$. Data are presented as the mean \pm SEM $(n=8)$. (D) Overexpression of Beclin-1 decreased the expression of c-Myc and p62 in sh-EBP50 cells. The tumor tissues were homogenized and the expressions of EBP50, Beclin-1, c-Myc or p62 were detected by Western blot. (E) Schematic diagram showing how EBP50 suppresses MCF-7 cell proliferation by promoting Beclin-1/p62-mediated lysosomal degradation of c-Myc.

(mTORC1), protects c-Myc against proteasome-mediated degradation, which suggests there is a strong correlation between the activation on autophagy and degradation of $\mathrm{c}-\mathrm{Myc}^{[21]}$. Luciana et al found that chaperone-mediated autophagy promotes c-Myc ubiquitination and degradation ${ }^{[22]}$ and our finding revealed that c-Myc is bound with p62 and degraded by macroautophagic lysosomal pathway directly. The above studies focused on the proteasome-mediated degradation of c-Myc and did not explored the autophagic lysosomal degra- dation of c-Myc. Hence, our studies revealed a novel degradation pathway of c-Myc by macroautophagic lysosomal pathway.

In summary, EBP50 up-regulates the lysosomal degradation of c-Myc and inhibits c-Myc-related signaling to suppress the multiplication of MCF-7 cells. Our findings define a novel signaling pathway and highlight the molecular mechanism used by EBP50 to mediate cancer cell proliferation. Based on our results, promoting the lysosomal degradation of c-Myc might 
be a promising new strategy for treating breast cancer.

\section{Abbreviations}

Cdc25A, cell division cycle 25 homolog A; CHX, cycloheximide; $\mathrm{CQ}$, chloroquine; EBP50, ezrin radixin moesin binding phosphoprotein-50; EGF, epidermal growth factor; ERK1/2, extracellular signal-regulated kinase 1 and 2; GST, glutathione $S$ transferase; NHERF, $\mathrm{Na}^{+} / \mathrm{H}^{+}$exchanger regulatory factor; NS, non-significant; mTORC1, mammalian target of rapamycin complex 1; SQSTM1, sequestosome 1; SRB, sulforhodamine B.

\section{Acknowledgements}

This work was supported by the National Natural Science Foundation of China (31401186, 81473249, 81321004 and 81673471), National Mega-project for Innovative Drugs (2014ZX09201042) and the CAMS Innovation Fund for Medical Sciences (CIFMS) (2016-I2M-2-002).

\section{Author contribution}

Hong LIU and Rong-guang SHAO designed research; Hong LIU and Wu-li ZHAO performed research; Jia-ping WANG and Bing-mu XIN contributed new reagents or analytic tools; Hong LIU and Jia-ping WANG performed animal experiment; Hong LIU and Rong-guang SHAO wrote the paper.

\section{Supplementary information}

Supplementary information is available on the website of Acta Pharmacologica Sinica.

\section{References}

1 Vaquero J, Nguyen Ho-Bouldoires TH, Claperon A, Fouassier L. Role of the PDZ-scaffold protein NHERF1/EBP50 in cancer biology: from signaling regulation to clinical relevance. Oncogene 2017; 36: $3067-$ 79.

2 Liu H, Ma Y, He HW, Wang JP, Jiang JD, Shao RG. SLC9A3R1 stimulates autophagy via BECN1 stabilization in breast cancer cells. Autophagy 2015; 11: 2323-34.

3 Yao W, Feng D, Bian W, Yang L, Li Y, Yang Z, et al. EBP50 inhibits EGF-induced breast cancer cell proliferation by blocking EGFR phosphorylation. Amino Acids 2012; 43: 2027-35.

4 Pan Y, Wang L, Dai JL. Suppression of breast cancer cell growth by $\mathrm{Na}^{+} / \mathrm{H}^{+}$exchanger regulatory factor 1 (NHERF1). Breast Cancer Res 2006; 8: R63.

5 Wade MA, Sunter NJ, Fordham SE, Long A, Masic D, Russell LJ, et al. c-MYC is a radiosensitive locus in human breast cells. Oncogene 2015; 34: 4985-94.

6 Green AR, Aleskandarany MA, Agarwal D, Elsheikh S, Nolan CC, DiezRodriguez M, et al. MYC functions are specific in biological subtypes of breast cancer and confers resistance to endocrine therapy in luminal tumours. Br J Cancer 2016; 114: 917-28.

$7 \mathrm{Xu}$ J, Chen Y, Olopade OI. MYC and breast cancer. Genes Cancer 2010; 1: 629-40.

8 Albajar M, Gomez-Casares MT, Llorca J, Mauleon I, Vaque JP, Acosta $\mathrm{JC}$, et al. MYC in chronic myeloid leukemia: induction of aberrant DNA synthesis and association with poor response to imatinib. Mol Cancer Res 2011; 9: 564-76.

9 Miller DM, Thomas SD, Islam A, Muench D, Sedoris K. C-Myc and cancer metabolism. Clin Cancer Res 2012; 18: 5546-53.

10 Herranz D, Maraver A, Canamero M, Gomez-Lopez G, Inglada-Perez L, Robledo M, et al. SIRT1 promotes thyroid carcinogenesis driven by PTEN deficiency. Oncogene 2013; 32: 4052-6.

11 Hubbard GK, Mutton LN, Khalili M, McMullin RP, Hicks JL, BianchiFrias D, et al. Combined MYC activation and Pten loss are sufficient to create genomic instability and lethal metastatic prostate cancer. Cancer Res 2016; 76: 283-92.

12 Liu H, Ma Y, He HW, Zhao WL, Shao RG. SPHK1 (sphingosine kinase 1) induces epithelial-mesenchymal transition by promoting the autophagy-linked lysosomal degradation of CDH1/E-cadherin in hepatoma cells. Autophagy 2017; 13: 900-13.

13 Yan LX, Wu QN, Zhang Y, Li YY, Liao DZ, Hou JH, et al. Knockdown of miR-21 in human breast cancer cell lines inhibits proliferation, in vitro migration and in vivo tumor growth. Breast Cancer Res 2011; 13: R2.

14 Zheng JF, Sun LC, Liu H, Huang Y, Li Y, He J. EBP50 exerts tumor suppressor activity by promoting cell apoptosis and retarding extracellular signal-regulated kinase activity. Amino Acids 2010; 38: 1261-8.

15 Zhang Y, Wang Z, Li X, Magnuson NS. Pim kinase-dependent inhibition of C-Myc degradation. Oncogene 2008; 27: 4809-19.

16 Jing $\mathrm{H}, \mathrm{Hu}$ J, He B, Negron Abril YL, Stupinski J, Weiser K, et al. A SIRT2-selective inhibitor promotes c-Myc oncoprotein degradation and exhibits broad anticancer activity. Cancer Cell 2016; 29: 767-8.

17 Yu Z, Li T, Wang C, Deng S, Zhang B, Huo X, et al. Gamabufotalin triggers c-Myc degra-dation via induction of WWP2 in multiple myeloma cells. Oncotarget 2016; 7: 1572537.

18 Pei Y, Yao Q, Yuan S, Xie B, Liu Y, Ye C, et al. GATA4 promotes hepatoblastoma cell proliferation by altering expression of miR125b and DKK3. Oncotarget 2016; 7: 77890-901.

19 Kang C, Xu Q, Martin TD, Li MZ, Demaria M, Aron L, et al. The DNA damage response induces inflammation and senescence by inhibiting autophagy of GATA4. Science 2015; 349: aaa5612.

20 Cianfanelli V, Fuoco C, Lorente M, Salazar M, Quondamatteo F, Gherardini PF, et al. AMBRA1 links autophagy to cell proliferation and tumorigenesis by promoting c-Myc dephosphorylation and degradation. Nat Cell Biol 2015; 17: 20-30.

21 Puustinen P, Rytter A, Mortensen M, Kohonen P, Moreira JM, Jaattela M. CIP2A oncoprotein controls cell growth and autophagy through mTORC1 activation. J Cell Biol 2014; 204: 713-27.

22 Gomes LR, Menck CFM, Cuervo AM. Chaperone-mediated autophagy prevents cellular transformation by regulating MYC proteasomal degradation. Autophagy 2017; 13: 928-40. 